\title{
DOROTA CZERKIES
}

Uniwersytet Jagielloński w Krakowie

e-mail: dorcz_92@interia.pl

\section{Une (en)quête du récit: Références au genre policier en tant que moyen d'un renouveau romanesque dans La Réticence de Jean-Philippe Toussaint}

\author{
Abstract \\ Searching for the Narrative Text: Narration and References to Detective \\ Fiction in the Novel Reticence by Jean-Philippe Toussaint
}

The aim of the article is to prove that the novel Reticence (1991) by Jean-Philippe Toussaint, Belgian writer, director and visual artist, is a parody of the detective novel's sub-genres: the mystery novel and the suspens novel. These references to popular literature, however, are not aimed only at producing the comical catharsis effect. On the contrary, they constitute especially the way of the revival of Gérard Genette's category of narrative discourse. The enigma, the suspens and the investigation, the predominant features of detective novels, shape the unique fictional universe, in which the author plays with his readers. Toussaint revives the novelistic genre on three levels: of the story, the narration and the narrative text. The mystery becomes the dominant element of the book, as well as a metaphor of the literary creation. In Reticence, Toussaint, as the omnipotent author, depicts his self-portrait to be able to discuss even better his own writing and the complex relations the book - the reader.

Keywords: Gérard Genette, narrative discourse, detective novel, Reticence, Jean-Philippe Toussaint.

Mots-clés: Gérard Genette, récit, genre policier, La Réticence, Jean-Philippe Toussaint.

Gérard Genette définit trois acceptions du terme «récit» de la façon suivante:

[...] il faut discerner nettement sous ce terme trois notions distinctes. Dans un premier sens - qui est aujourd'hui, dans l'usage commun, le plus évident et le plus central -, récit désigne l'énoncé narratif, le discours oral ou écrit qui assume la relation d'un événement ou d'une série d'événements [...]. Dans un second sens, moins répandu, mais aujourd'hui courant chez les analystes et théoriciens du contenu narratif, récit désigne la succession d'événements, réels ou fictifs, qui font l'objet de ce discours, et leurs diverses relations 
[...] un ensemble d'actions et de situations considérées en elles-mêmes, abstraction faite du médium [...]. En un troisième sens qui est apparemment le plus ancien, récit désigne encore un événement: non plus toutefois celui que l'on raconte, mais celui qui consiste en ce que quelqu'un raconte quelque chose: l'acte de narrer pris en lui-même'.

Cette définition permet à Genette de proposer respectivement trois catégories: récit proprement dit, histoire et narration ${ }^{2}$. Nombre de critiques attribuent un particulier retour du $(\mathrm{au})$ récit aux auteurs qui, à la charnière des années 70 . et 80 . du $\mathrm{XX}^{\mathrm{e}}$ siècle, ont débuté dans Les Editions de Minuit. Ce groupe d'écrivains est néanmoins loin d'être qualifié avec une dénomination univoque. Au contraire, on leur colle plusieurs étiquettes: «clan, génération, vague, tendance, nébuleuse, romanciers impassibles», pourtant - jamais «mouvement». Tout un inventaire de traits caractéristiques de leur écriture est cité: désinvolture, incongruité, minimalisme, réduction de la narration, tendance à la réécriture, caractère ludique, banalité ou encore présence de litote. Il est à observer, avec Fieke Schoots, que cette pluralité de perspectives rend le groupe encore moins homogène ${ }^{3}$.

D'après Jacques Pierre Amette, Jean-Philippe Toussaint est le porte-drapeau de ce groupe de «nouveaux nouveaux romanciers». D'ailleurs, c'est le premier roman toussaintien paru en forme papier, La Salle de bain, qui a donné le nom à ces écrivains aux yeux de Lucie Vermij - «la génération de 'salle de bain'»" Il est pourtant à constater que Toussaint, écrivain, plasticien et cinéaste belge, a développé son propre style tout en remettant en question l'écriture et le rôle de l'écrivain ${ }^{5}$, ainsi que le récit dans son acception événementielle et narrative.

Dans cet article, nous entendons prouver que, contre le chaos postmoderne ${ }^{6}$, dans le roman intitulé La Réticence, Jean-Philippe Toussaint tente d'opérer un renouveau du romanesque, et cela à l'aide de recours au genre policier.

\section{Littérature populaire et roman policier}

Dans un des entretiens qui accompagnaient la parution de la tétralogie romanesque toussaintienne, «Marie Madeleine Marguerite de Montalte», l'écrivain belge a semblé implicitement fasciné par la littérature populaire:

Une autre façon pour moi de mettre cet amour à l'épreuve est d'intégrer des éléments qui s'apparentent au roman policier, comme tout ce qui concerne l'épisode de l'incendie criminel de la chocolaterie dans Nue. Il y a là un énorme pan secret du livre, enfoui, non divulgué, qui est comme la partie invisible de sa structure. C'est la même chose avec l'épisode du trafic de drogue dans Fuir, qui n'est jamais explicitement abordé, mais qui renforce la dramatisation du récit. J'attache en général une grande importance aux détails roman-

1 G. Genette, Figures III, Paris 1972, p. 71.

2 Ibid., p. 72.

3 F. Schoots, „, Passer en douce à la douane”. L'Ecriture minimaliste de Minuit: Deville, Echenoz, Redonnet et Toussaint, Amsterdam-Atlanta 1997, p. 10-11, 15-18, 49-50.

4 Ibid., p. 16-17.

5 F. Wagner, Monsieur Jean-Philippe Toussaint et la notion de Vérité. Pour une poétique perspectiviste, «Textyles» 2010, no. 38.

${ }^{6}$ F. Schoots, op. cit., p. 128-137. 
esques, qui peuvent s'apparenter à ce qu'au cinéma on appelle les accessoires. [...] il faut [...] partir d'un élément concret, ponctuel, qui sera porteur de significations qui le dépassent. Il y a toujours, dans mes livres, la présence d'éléments inquiétants, parfois très simples [...] ils participent au plaisir de la lecture, au suspense [...] aussi, ils créent un contexte d'insécurité, d'inquiétude $[\ldots]^{7}$.

Le roman populaire est un espace de concurrence entre le bien et le mal, entre le «Héros» et le «Justicier». Profondément enraciné dans les circonstances sociales du XIX $\mathrm{XI}^{\mathrm{e}}$ siècle, ce type de roman est loin d'être homogène à l'égard des sous-genres. Toutefois, il est traîté comme une forme inférieure de littérature ${ }^{8}$. Jacques Migozzi note trois acceptions de la notion de «littérature populaire»: littérature orale, folklorique, littérature de colportage et roman populaire «du XIX ${ }^{\mathrm{e}}$ siècle et de la Belle Epoque» ${ }^{9}$. Parmi les genres populaires, selon Geneviève Bollème, il est à énumérer: littérature de colportage sur justice et vengeance, contes merveilleux et fantastiques, romans gothiques, romans sentimentaux, histoires sur brigands, récits historiques ${ }^{10}$. Marc Lits y ajoute encore roman noir, roman de terreur, mélodrame et roman-feuilleton ${ }^{11}$.

C'est précisement grâce aux romans-feuilletons, romans gothiques, mélodrames, ou encore faits divers journalistiques qu'a émergé le roman policier ${ }^{12}$. Il est, pourtant, comme toute littérature populaire, un phénomène complexe. Aussi, évolue-t-il constamment ${ }^{13}$.

\section{Sous-genres policiers}

Marc Lits propose une liste de dix raisons pour lesquelles le roman policier est digne d'attention et de (re)lecture. Les écrits policiers suscitent donc chez le lecteur un plaisir qui relève des images émotionnelles, du suspense et de la structure narrative caractéristique. La paralittérature encourage aussi des «non-lecteurs» à essayer de lire un texte plus accessible. Les récits policiers touchent des questions essentielles, surtout celle de la mort. Ils permettent de voir le fond sociologique de littérature, les relations entre le littéraire et le réel. De plus, le genre policier reflète clairement les structures de tous les textes romanesques ${ }^{14}$. Jean Tortel résume tout récit populaire dans la formule de «la recherche de domination, toute-

7 J. Garcin, Je suis très connu, mais personne ne le sait [dans:] J.-Ph. Toussaint, M.M.M.M., entretiens littéraires, Paris 2017, p. 4-5.

8 J. Tortel, Le roman populaire [dans:] Entretiens sur la paralittérature, dir. N. Arnaud, F. Lacassin, J. Portel, Cerisy-la-Salle 1970, p. 55-70.

9 J. Migozzi, Boulevards du populaire, Limoges 2005, p. 25-29.

10 G. Bollème, La bibliothèque bleue, «Archives» 1971, no. 44, cité selon M. Lits, Le roman policier: introduction à la théorie et à l'histoire d'un genre littéraire, Liège 1993, p. 24.

11 M. Lits, Le roman ..., p. 24-26.

12 Ibid., p. 37.

13 A.-M. Thiesse, Mauvais genres: quelques réflexions sur la notion de lecture populaire, «Pratiques» 1987, no. 54, cité selon M. Lits, Le roman ..., p. 23.

14 M. Lits, Le genre policier dans tous ses états. D'Arsène Lupin à Navarro, Limoges 2011, p. 13-14. 
puissance» ${ }^{15}$. Le roman policier en constitue une partie, celle qui se base, selon Charles Grivel, sur la présence indispensable d'éléments suivants: la trinité Abel - Caïn - Dieu, un «jeu combinatoire» sur lequel le texte se base, l'ignorance de la part du lecteur, une logique spécifique, une dimension mythique et rituelle. En effet, dans la fiction policière on a affaire à un méchant criminel, une victime et un bon détective-justicier. Le narrateur est ouvertement présent. Il commente ce dont il est témoin. L'auteur, lui, joue à être témoin. Le bien et le mal luttent l'un contre l'autre au grand plaisir du lecteur qui ne souhaite connaitre la solution d'énigme que le plus tard possible. Le roman policier revendique sa vraisemblance, pour pouvoir mieux favoriser un mythe au service d'un groupe concret. Le rituel de l'épouvante, le terrifiant et l'émotion s'installent. Le lecteur, pour son plaisir, assiste à «un spectacle, un exorcisme, un acte collectif d'épuration» qui ont pour but de l'instruire ${ }^{16}$.

Lits caractérise le roman policier en ayant recours au titre de la traduction française d'une nouvelle d'Edgar Allan Poe. Charles Baudelaire a traduit The murders in the rue Morgue par Double assassinat dans la rue Morgue. Ainsi, selon Lits, on peut discerner quatre aspects clés de toute fiction policière: la double structure, le sujet criminel, l'enracinement dans la réalité et la présence d'un imaginaire lié à la mort. La fiction policière présente deux histoires: celle du crime et celle de la vengeance sur le méchant. On essaie de resourdre une énigme à rebours - à travers une retrospection. Un meutre et la présence de la police sont indispensables. Dans l'histoire de l'enquête le raisonnement logique joue le rôle crucial. D'après Lits, la fascination pour le roman policier découle de son «imaginaire mortuaire, terreur ancestrale, peur face au crime, au sang, à la mort». Le livre nous fait frissonner pour pouvoir expliquer le mystère ${ }^{17}$.

Le roman policier, «le récit d'enquête», donne la fonction prédominante ${ }^{18}$ au «code herméneutique» que Roland Barthes définit de la façon suivante:

$[\ldots]$ l'ensemble des unités qui ont pour fonction d'articuler, de diverses manières, une question, sa réponse et les accidents variés qui peuvent ou préparer la question ou retarder la réponse; ou encore: de formuler une énigme et d'amener son déchiffrement. [...] termes (formels), au gré desquels une énigme se centre, se pose, se formule, puis se retarde et enfin se dévoile ${ }^{19}$.

Jacques Dubois souligne à cet égard que le récit policier est celui qui «ne trouve plus sa finalité qu'en lui-même» ${ }^{20}$. Il est à constater que l'on peut trouver, avec Régis Messac, des prédécesseurs du genre policier en Antiquité, ainsi qu'au $\mathrm{XVIII}^{\mathrm{e}}$ siècle $^{21}$. Certes, il y a des point communs considérables entre le genre

15 J. Tortel, op. cit., p. 64, 67.

16 Ch. Grivel, Observation du roman policier [dans:] Entretiens sur ..., p. 232-247.

17 M. Lits, Le roman ..., p. 75-88.

18 J. Dubois, Indicialité du récit policier [dans:] Narration et interprétation, dir. C. Gothot-Mersch, Bruxelles 1984, p. 116, cité selon M. Lits, Le genre policier ..., p. 24.

19 R. Barthes, S/Z, Paris 1970, p. 24, 26, cité selon M. Lits, Le genre policier ..., p. 24.

20 J. Dubois, op. cit., p. 116, cité selon M. Lits, Le genre policier ..., p. 24.

21 R. Messac, Le «detective novel» et l'influence de la pensée scientifique, Paris 1929, Genève 1975, p. 160, cité selon M. Lits, Le roman..., p. 32. 
policier et la tragédie antique. Pourtant, les premiers textes policiers ont apparu sous la plume d'Edgar Allan Poe. C'étaient des nouvelles fortement imprégnées du fantastique, accentuant donc le mystère, l'inexplicable. En France, la production littéraire d'Emile Gaboriau est digne de souligner à cet égard ${ }^{22}$. Lits qualifie le roman policier de «genre protéiforme». En effet, parmi ses sous-genres on peut distinguer: récit d'énigme dit classique, identifiable au roman jeu, roman d'espionnage, roman noir ou série noire, roman de la peur ou de la victime, thriller, récit de suspense et néo-polar qui dénonce les vices du pouvoir politique ${ }^{23}$. L'auteur cité met en valeur surtout le récit d'énigme ou «roman à énigme» todoro$\operatorname{vien}^{24}$.

Les sous-genres policiers respectifs se laissent différencier selon leurs dominantes, donc selon «Les traits du genre, c'est-à-dire des procédés qui organisent la composition de l'œuvre $[\ldots]$ procédés dominants $[\ldots]$ tous les autres procédés nécessaires à la créationde l'ensemble artistique leur sont soumis» ${ }^{25}$. Dans le roman noir l'élément crucial est l'action, le roman de suspense exploite le mystère, la tension et «l'histoire du suspect-détective» ${ }^{26}$. Pour le roman d'espionnage, c'est l'aventure qui règne, le polar et le néo-polar, eux, mettent l'accent respectivement sur la violence et la description sévère des gens au pouvoir et de la société27.

D'après François Fosca, le récit d'énigme, identifiable au «roman-problème, roman de pure détection, roman de détection criminelle», doit être écrit selon quelques règles immuables. Il s'agît d'expliquer un mystère à première vue inexplicable. Plusieurs personnages sont successivement et à tort considérés coupables. Le raisonnement n'est pas à-prioriste, mais basé sur la logique et sur l'observation des faits. Les cas les plus difficiles s'avèrent les plus faciles à expliquer. La solution correcte est basée sur les faits mais imprévue tout au long de l'histoire. Pourtant, toutes les voies impossibles éliminées, il ne reste qu'elle. Le mystère est resolu par un amateur, non pas par un policier. Le narrateur, lui, sait moins que ce détective-amateur et il en est conscient. Pourtant, il ne lui en veux $\operatorname{pas}^{28}$. Roger Caillois souligne que, conçu ainsi, surtout par les auteurs anglo-saxons, le roman est devenu «un exercice d'esprit», il s'est rapproché de la science et des jeux de société2 ${ }^{2}$. Lits trouve que ce roman est «roman-jeu [...] [dans lequel] le romancier jouera contre le lecteur, lui donnant des indices en cours de lecture

22 M. Lits, Le roman ..., p. 87, 129-133.

23 M. Lits, Le genre policier ..., p. 27-30.

24 M. Lits, Le roman ..., op. cit., p. 57.

25 B. Tomachevski, Les genres littéraires [dans:] idem, Théorie de la littérature, Paris 1965, p. 303, cité selon M. Lits, Le roman ..., p. 19.

26 T. Todorov, Typologie du roman policier [dans:] idem, Poétique de la prose, Paris 1971, p. 63-64, cité selon M. Lits, Le roman ..., p. 60.

27 M. Lits, Le roman ..., p. 56-63.

28 F. Fosca, Histoire et technique du roman policier, Paris 1937, p. 62-63, cité selon M. Lits, Le roman ..., p. 43-44.

29 R. Caillois, Puissances du roman [dans:] idem, Approches de l'imaginaire, Paris 1974, p. 193, cité selon M. Lits, Le roman ..., p. 44-45. 
pour lui permettre de découvrirle coupable avant que le dernier chapitre ne révèle la bonne solution» ${ }^{30}$.

Le récit d'énigme est une forme de devinette. Deviner ou poser une devinette indéchiffrable, écrit André Jolles, signifie «la vie». Qui ne sait pas deviner, échoue. Le récit d'énigme n'est pas la solution mais sa quête. Les questions et les réponses luttent les unes avec les autres. L'accès au savoir est le facteur qui rend certains protagonistes criminels sympathiques et des détectives enquêteurscriminels $^{31}$. Claude-François Ménestier souligne que le caractère énigmatique du récit est un trait crucial de chaque enquête fictionnelle, en donnant le plaisir aux lecteurs ${ }^{32}$. Sigmund Freud, lui, trouve que le criminel et l'hystérique se comportent d'une façon analogue ${ }^{33}$. Le récit d'énigme donne du plaisir grâce aux signes, aux indices, vraies et fausses, qu'il faut bien déchiffrer et qui favorisent une lecture détournée ${ }^{34}$. Le récit d'énigme était exploré par plusieurs écrivains, comme nouveaux romanciers ou oulipiens ${ }^{35}$. Jean-Philippe Toussaint exploite le récit d'énigme et le roman de suspense, des sous-genres policiers ${ }^{36}$, à ses propres fins.

\section{Récit réticent}

La Réticence, roman paru en 1991 aux Editions de Minuit, est l'histoire d'un homme de trente trois ans qui vient dans un petit village de Sasuelo avec son fils âgé de huit mois. Il est le narrateur homodiégétique ${ }^{37}$. Il narre les faits à la première personne. Triparti, le roman commence par la découverte d'un chat mort dans les eaux du port de Sasuelo. Cette image devient, peu à peu, un élément obsédant le narrateur. En arrivant à Sasuelo, le protagoniste a pour but de visiter ses amis, les Biaggi. Néanmoins, il évite constamment cette visite. Finalement, elle ne se réalise jamais. Ce roman que Jean-Philippe Toussaint a dédié à sa femme Madeleine, revisite les dominantes du récit d'énigme et du roman de suspense une énigme à resoudre, des pistes à déchiffrer, le mystère et la tension, le motif du suspect-détective ${ }^{38}$. La mise en valeur de ces motifs policiers s'opère à tous les trois niveaux genettiens.

30 M. Lits, Le roman ..., p. 46.

31 Ibid., p. 92 - 93; A. Jolles, Formes simples, Paris 1972, p. 119, 108, 109, 117, cité selon M. Lits, Le roman ..., p. 91-93.

32 C.-F. Ménestier, La philosophie des images énigmatiques, repoublié partiellement sous le titre Poétique de l'énigme, «Poétique»1981, no. 45, p. 31, 36, 37, 40, cité selon M. Lits, Le roman..., p. 93-94.

33 S. Freud, La psychanalyse et l'établissement des faits en matière judiciaire par une méthode diagnostique [dans:] idem, Essais de psychanalyse appliquée, Paris 1971, p. 51, cité selon M. Lits, Le roman..., p. 98.

34 M. Lits, Le roman ..., p. 99-100.

35 Ibid., p. 108.

36 S. Glasco, Parody and palimpsest. Intertextuality, language and the ludic in the novels of Jean-Philippe Toussaint, New York 2015, p. 100.

37 G. Genette, Figures III, p. 75; cf. idem, Récit fictionnel, récit factuel, «Protée» 1991, no. 19(1), p. $12-15$.

38 J.-Ph. Toussaint, La Réticence, Paris 1991, p. 7, 11-159. 


\section{Histoire}

L'action du livre se déroule dans un petit village situé sur une île ${ }^{39}$. Un tel lieu d'action favorise l'impression de solitude chez le protagoniste-narrateur. Sarah Glasco associe cette île à la Corse qui, tout comme la Sardaigne italienne, suscite des connotations avec le crime organisé. L'ami du narrateur qui vit à Sasuelo, a aussi un nom corse, Biaggi. Le lecteur ne sait pas qui sont les Biaggi, pourquoi ils sont absents, pour quelles raisons le protagoniste veut voir son ami et pourquoi, finalement, il rénonce à sa démarche. L'obscur, l'insolite et l'absurde règnent dans cette histoire. Une telle ambiance croît encore à cause d'omniprésence des couleurs sombres, dont le gris se détache le plus. L'obscurité et le silence créent une tension et favorisent l'attente de ce qui va se passer. Le narrateur est un drôle de détective qui veut connaître le pourquoi de la mort du chat noir trouvé dans le port. Néanmoins, il ne peut pas l'expliquer parce qu'il est de plus en plus désorienté. Le motif du chat noir mort peut faire penser à une nouvelle de Poe, The Black Cat. Un autre chat noir, d'ailleurs, perturbe aussi la paix du narrateur de La Réticence sur une route du village. Les fausses pistes sont de plus en plus nombreuses. Le protagoniste ne sait plus si quelqu'un a tué le chat, si c'était Biaggi, ou si c'était son ami qui a été assassiné. Le narrateur se sent surveillé par un homme dans une Mercedes grise. Il vole, pour des raisons inconnues, sa propre lettre de la boîte postale des Biaggi. L'ambiance de l'ambiguïté approfondit encore sa paranoïa. Le mystère se réduit à un cliché encore plus banal. Il se passe beaucoup de choses, le suspense est hyperbolique. L'histoire est en train de se construire, le lecteur observe la recherche d'une histoire ${ }^{40}$. Le protagoniste-narrateur est, tour à tour, un détective-amateur et un assassin ${ }^{41}$.

\section{Narration}

Le narrateur de La Réticence est un homme de trente trois ans qui reste à Sasuelo pendant sept jours. Ce détail permet à Glasco d'évoquer des connotations avec Jésus Christ, crucifié à même âge, et, en même temps, avec Dieu, qui a créé notre monde en six jours pour pouvoir en suite se réposer pendant le septième ${ }^{42}$. Il est à constater que cet élément biblique contraste fortement avec le caractère paranoïaque de l'enquête poursuivie par le protagoniste. Glasco formule l'opinion selon laquelle le saugrenu, le drôle et l'humour vulgaire sont pratiquement absents de La Réticence si l'on compare ce roman avec les précédents. Pourtant, selon l'auteure, la parodie du roman d'énigme et le comportement du narrateur,

\footnotetext{
39 Ibid., p. 159.

40 R. Caldwell, Jean-Philippe Toussaint [dans:] The contemporary novel in France, dir. W. Thompson, Gainesville 1995, p. 369, cité selon S. Glasco, op. cit., p. 98.

41 S. Glasco, op. cit., p. 95-118.

42 Ibid., p. 103.
} 
doté d'un caractère comique, sont, pour les lecteurs, une source considérable de l'effet de catharsis $^{43}$.

Selon Ruth Amar, la réticence, la aposiopesis, figure rhétorique basée sur l'omission et l'interruption, constitue une stratégie narrative toussaintienne, et avant tout, dans le roman éponyme. Cette réticence se manifeste par l'inexplicable, la narration fragmentée et répétitive, «des silences, des non-dits, des arrêts de narration». Les phrases sont souvent inachevées, elliptiques, lacunaires, rompues. Et pourtant, ce sont précisément ces traits caractéristiques de la narration qui favorisent, grâce à la polysémie, la construction de l'histoire, ou des histoires, devant les yeux du lecteur de La Réticence. L'absence apparente de l'histoire devient en fait une présence-absence du narrateur ${ }^{44}$. Comme le constate Gianfranco Rubino, le narrateur du roman en question est actif, malgré la stagnation narrative apparente et la répétition des détails banals. Le protagoniste s'apperçoit de ce qui se passe mais aussi de ce qui ne se passe pas - le silence est aussi important que le manque des sons. L'action, ainsi que la non-action, sont mises en valeur, grâce à une narration et une narrativité paradoxales ${ }^{45}$.

Le narrateur-protagoniste est un détective dépourvu de la capacité de capter et de lire correctement les signes. Il est hanté par quelques images récurrentes. Son regard déforme la réalité, tous les objets qui l'entourent. Ce personnage n'est pas capable de voir son propre reflet dans le miroir. Au contraire, seul un autre regard persévérant s'y détache, celui qui surveille constamment le protagoniste. Ce dernier est comme son fils, comme un petit enfant, ne sachant pas déchiffrer les signes qu'il essaie pourtant de comprendre et d'interpréter ${ }^{46}$.

Glasco constate que le narrateur de La Réticence essaie de conduire une enquête basée sur un raisonnement logique et une recherche persévérante des causes des faits, mais, malgré son effort, il revient constamment à son point de départ. Malgré la présence des analepses et des retours narratifs, le protagoniste est obsédé par la mort du chat et la disparition de Paul Biaggi. Le narrateur pose tout le temps les mêmes questions. En réponse à lui-même, il formule ses propres hypothèses et théories, qui, pourtant, ne le convainquent que pour un moment. En voulant formuler des constatations fiables, il raisonne et se rassure sans cesse. Mais, ne se donne-t-il que des informations qui brouillent les pistes. Il utilise des expressions et tournures comme «en quelque sorte, car, se pouvoir». Voulant justifier ses théories, il ne fait que croître ses doutes, son angoisse et l'ambiance d'incertitude, en formulant des phrases interrogatives, inachévées, avec des points de suspension. Au niveau de la grammaire, le narrateur se sert du plus-que-parfait avec «certainement, sûr, auparavant, peut-être», du conditionnel et des verbes comme «sembler, pouvoir, devoir» au lieu d'«être». La réticence et, en même temps, l'obsession du narreteur-protagoniste favorisent la construction de l'histoire, qui n'est pas

43 Ibid., p. 96, 97, 100-103.

44 R. Amar, Dispositifs minimalistes ou réticents? [dans:] Les vérités de Jean-Philippe Toussaint, dir. S. Chaudier, Saint-Etienne 2016, p. 191-199.

45 G. Rubino, Jean-Philippe Toussaint: une narrativité paradoxale [dans:] Romanciers minimalistes 1979-2003, dir. M. Dambre, B. Blanckeman, Paris 2012, p. 71-80.

46 F. Schoots, op. cit., p. 126-127, 135. 
absente du livre mais cachée. Aussi, favorisent-elles l'usage ludique du suspense, du mystère, de l'énigme. Elles sont, enfin et surtout, la réticence et l'obsession de l'auteur, de l'écrivain ${ }^{47}$.

\section{Récit proprement dit}

Jean-Philippe Toussaint parodie ainsi le récit d'énigme et le roman de suspense. Mais, ne le fait-il pas que pour assurer l'effet comique ${ }^{48}$. En poursuivant, avec et contre le protagoniste, son enquête parodique, il effectue la «quête d'un récit». Les personnages comme les Biaggi, écrit Amar, complottent avec l'auteur contre le narrateur. Or tous les protagonistes toussaintiens constituent «plutôt des personnages expérimentaux dans le laboratoire de l'auteur $»^{49}$. L'auteur et le protagoniste demeurent réticents pour, à la fois, retarder et faire avancer le récit, le texte narratif ${ }^{50}$.

L'écrivain belge situe l'action de La Réticence en Corse, où il vivait à l'époque de la création du livre ${ }^{51}$. Il le dédie à sa femme ${ }^{52}$. L'œuvre marque non seulement un moment spécial dans la vie de Jean-Philippe Toussaint, celui de la naissance de son premier enfant, d'un garçon ${ }^{53}$ mais elle est avant tout un signe du changement significatif dans l'écriture de l'auteur belge. Dans L'Urgence et la Patience il l'explique ainsi:

Jusqu'à La Réticence, quand j'entreprenais un livre, je travaillais tous les jours [...] sans reprendre haleine [...] jusqu'à un an d'affilée. Je considérais l'écriture comme une machinerie lourde qui se mettait en place sur la longue durée, quelque chose de régulier, de pesant, d'entravé, $[\ldots]$ qui avançait péniblement, pouce à pouce - une charrue. L'expérience douloureuse de l'écriture de La Réticence, livre que je n'arrivais pas à écrire,que j'ai failli abandonner plusieurs fois [...] m'a amené à décider de ne plus jamais écrire de cette manière [...] je n'ai plus travaillé que porté par un élan, pendant des sessions d'écriture [...] de quinze jours à trois mois maximum, entrecoupées de longues périodes où je faisais autre chose $[\ldots]$ où je vivais $[\ldots]^{54}$.

Glasco observe que La Réticence reflète, à travers la paranoïa du narrateur, tous les dilemmes, les doutes et les questions qu'éprouve l'auteur face à l'écriture d'un nouveau livre. L'attitude ambivalente et les émotions contradictoires du protagoniste à l'égard de son fils, figurent clairement les relations complexes entre

47 S. Glasco, op. cit., p. 99-118.

48 Ibid., p. 99-103.

49 R. Amar, op. cit., p. 196, 197. L'Auteure évoque ici des constatations de Warren Motte. Cf. W. Motte, Reading Jean-Philippe Toussaint, «Context, a forum for literary art and culture», no. 12, online edition, http://www.centerforbookculture.org/context/no12/Motte.html (accès: 15.02.2019).

50 F. Schoots, op. cit., p. 127.

51 S. Glasco, op. cit., p. 97.

52 J.-Ph. Toussaint, La Réticence, p. 7.

53 J.-Ph. Toussaint, Le jour ou j’ai commencé La Réticence, «www.bon-a-tirer.com. Revue littéraire en ligne» 2002, no. 6, http://www.bon-a-tirer.com/volume6/jpt.html (accès: 15.02.2019).

54 J.-Ph. Toussaint, L'Urgence et la Patience [dans:] L'Urgence et la Patience, Paris 2012, p. $21-22$. 
l'écrivain et son texte, entre la fiction et la vie, entre le littéraire et le réel. La réticence devient le mode et la métaphore de la création ${ }^{55}$.

Schoots argumente qu'il n'y a point d'enquête:

D'abord, le narrateur est incapable de mener à bien son enquête. Sa paranoïa l'empêche de bien interpréter les signes et de trouver des réponses aux questions soulevées. Ensuite, il n'y a pas de véritable dénouement, de sorte que nombre de faits restent inexpliqués. En termes barthésiens: une énigme a été formulée mais son déchiffrement n'a pas été amené. Faute d'une victime, il n'y a même pas au de crime. La question initiale était donc mal posée. L'enquête ne fut après tout qu'une chimère ${ }^{56}$.

Pourtant, on peut constater, avec Amar, que l'énigme et l'enquête ne sont pas absentes de La Réticence. Cachées de premier abord, elles sont créées grâce à la réticence. Celle-ci forme un univers qui donne de la liberté à l'auteur ainsi qu'au lecteur. Ce dernier, souligne Toussaint, demeure en «relation importante» avec le livre à travers «une alchimie secrète» qu'est la lecture ${ }^{57}$. Glasco argumente, de surcroît, que l'univers fictif toussaintien favorise plusieurs lectures et interprétations $^{58}$. Jean-Philippe Toussaint effectue une «narrativisation» du texte grâce au retour à «l'histoire, au roman, à l'imaginaire» ${ }^{59}$.

Glasco met en valeur la présence des figures de l'écrivain et de son texte dans La Réticence, sous les traits du narrateur-protagoniste et ceux de son fils. L'auteur est présent dans le texte narratif et regarde constamment le narrateur. Il profite volontiers de son imagination ${ }^{60}$. Il se sert sans cesse des motifs du roman-jeu pour mener son propre jeu avec le lecteur. Il construit le «récit d'absence» et non pas un «récit absent» ${ }^{61}$. Plein d'ellipses typographiques, narratives et événementielles, La Réticence est un roman qui laisse au lecteur un espace considérable pour l'interprétation de l'angoisse du narrateur et de l'auteur ${ }^{62}$.

La Réticence, avec son jeu sur l'énigme et le suspense policiers, s'inscrit dans la poétique toussaintienne de l'autoportrait que l'écrivain belge postule dans L'Autoportrait (à l'étranger):

[...] En peinture, il y a toujours quelque chose d'émouvant dans la pratique de l'autoportrait, d'immédiatement bouleversant, d'essentiellement humain. Dans ses derniers autoportraits, Rembrandt nous regarde avec fixité, coiffé d'un simple mouchoir [...] Quand Rembrandt peint des autoportraits, c'est de peinture qu'il nous parle, pas de lui-même. Certes, il se prend lui-même comme sujet d'étude, mais sa personne n'est qu'un prétexte, c'est son art qu'il interroge. [...] il m'est également arrivé de me "peindre» au détour d'un paragraphe $[\ldots]$ petit autoportrait, tableautin ou vignette, une miniature qui obéirait aux règles

55 S. Glasco, op. cit., p. 103-118.

56 F. Schoots, op. cit., p. 135.

57 R. Amar, op. cit., p. 196, 197. Cf. L'entretien de Jean-Louis Tallon avec Toussaint en Bruxelles en 2002, http://perso.orange.fr/erato/horspress/toussaint.htm (accès: 15.02.2019).

58 S. Glasco, op. cit., p. 102.

59 A. Kibédi Varga, Le récit postmoderne, «Littérature» 1990, no. 77, p. 16, cité selon F. Schoots, op. cit., p. 101.

60 S. Glasco, op. cit., p. 97-118.

${ }_{61}$ R. Amar, op. cit., p. 194.

${ }^{62}$ F. Schoots, op. cit., p. 113-114. 
immuables du genre [...] aux règles que je n'avais jamais édictées, et que je n'écrivais généralement pas $^{63}$.

L'Auteur ne laisse pas que des «traces» dans son texte ${ }^{64}$. Au contraire, il y est constamment présent pour créer son «autoportrait oblique» romanesque ${ }^{65}$. Le suspense et l'énigme, grâce à la réticence, deviennent une métaphore du processus de création littéraire ${ }^{66}$. Dans l'univers toussaintien, comme le constate Amar, le lecteur et l'auteur sont tout-puissants - il peut s'y passer de tout. Le lecteur y mene sa propre enquête ${ }^{67}$. Parallèlement à celle-ci, toujours actif, l'auteur est en quête constante d'une «énergie romanesque pure»" ${ }^{68}$, d'une énergie «invisible, brûlante et presque électrique [...] qui fait qu'à un moment la pupille du lecteur se dilate» ${ }^{69}$.

\section{Bibliographie}

Amar R., Dispositifs minimalistes ou réticents? [dans:] Les vérités de Jean-Philippe Toussaint, dir. S. Chaudier, Saint-Etienne 2016.

Barthes R., S/Z, Paris 1970.

Barthes R., Sade, Fourier, Loyola, trad. R. Lis, Warszawa 1996.

Bollème G., La bibliothèque bleue, «Archives» 1971, no. 44.

Bourmeau S., Ecrivain contemporain [dans:] J.-Ph. Toussaint, M.M.M.M., entretiens littéraires, Paris 2017.

Caillois R., Puissances du roman [dans:] idem, Approches de l'imaginaire, Paris 1974.

Caldwell R., Jean-Philippe Toussaint [dans:] The contemporary novel in France, dir. W. Thompson, Gainesville 1995.

Desplechin M., Je cherche une énergie romanesque pure [dans:] J.-Ph. Toussaint, M.M.M.M., entretiens littéraires, Paris 2017.

Dubois J., Indicialité du récit policier [dans:] Narration et interprétation, dir. C. Gothot-Mersch, Bruxelles 1984.

Fosca F., Histoire et technique du roman policier, Paris 1937.

Freud S., La psychanalyse et l'établissement des faits en matière judiciaire par une méthode diagnostique [dans:] idem, Essais de psychanalyse appliquée, Paris 1971.

Garcin J., Je suis très connu, mais personne ne le sait [dans:] J.-Ph. Toussaint, M.M.M.M., entretiens littéraires, Paris 2017.

Genette G., Figures III, Paris 1972.

Genette G., Récit fictionnel, récit factuel, «Protée» 1991, no. 19(1).

63 J.-Ph. Toussaint, L'Autoportrait (à l'étranger), Paris 2000/2012, p. 10-14.

64 R. Barthes, Sade, Fourier, Loyola, trad. R. Lis, Warszawa 1996, cité selon A. Zawadzki, Autor. Podmiot literacki [dans:] Kulturowa teoria literatury. Główne pojęcia i problemy, dir. M.P. Markowski, R. Nycz, Kraków 2012, p. 241-242.

65 J.-B. Vray, L'Autoportrait ludique ou le bonnet noir de la mélancolie [dans:] Les Vérités..., p. 159.

66 S. Glasco, op. cit., p. 95-96, 103-118.

67 R. Amar, op. cit., p. 196-198.

68 M. Desplechin, Je cherche une énergie romanesque pure [dans:] J.-Ph. Toussaint, M.M.M.M...., p. 27.

69 S. Bourmeau, Ecrivain contemporain [dans:] J.-Ph. Toussaint, M.M.M.M...., p. 22. 
Glasco S., Parody and palimpsest. Intertextuality, language and the ludic in the novels of Jean-Philippe Toussaint, New York 2015.

Grivel Ch., Observation du roman policier [dans:] Entretiens sur la paralittérature, dir. N. Arnaud, F. Lacassin, J. Portel, Cerisy-la-Salle 1970.

Jolles A., Formes simples, Paris 1972.

Kibédi Varga A., Le récit postmoderne, «Littérature» 1990, no. 77.

Lits M., Le genre policier dans tous ses états. D’Arsène Lupin à Navarro, Limoges 2011.

Lits M., Le roman policier: introduction à la théorie et à l'histoire d'un genre littéraire, Liège 1993.

Ménestier C.-F., La philosophie des images énigmatiques, repoublié partiellement sous le titre Poétique de l'énigme, «Poétique»1981, no. 45.

Messac R., Le «detective novel» et l'influence de la pensée scientifique, Paris 1929, Genève 1975.

Migozzi J., Boulevards du populaire, Limoges 2005.

Motte W., Reading Jean-Philippe Toussaint, «Context, a forum for literary art and culture», no. 12, online edition, http:/www.centerforbookculture.org/context/ no12/Motte.html (accès: 15.02.2019).

Rubino G., Jean-Philippe Toussaint: une narrativité paradoxale [dans:] Romanciers minimalistes 1979-2003, dir. M. Dambre, B. Blanckeman, Paris 2012.

Schoots F., «Passer en douce à la douane». L'Ecriture minimaliste de Minuit: Deville, Echenoz, Redonnet et Toussaint, Amsterdam-Atlanta 1997.

Tallon J.-L., Entretien avec J.-Ph. Toussaint en Bruxelles en 2002, http://perso.orange. fr/erato/horspress/toussaint.htm (accès: 15.02.2019).

Thiesse A.-M., Mauvais genres: quelques réflexions sur la notion de lecture populaire, «Pratiques»1987, no. 54.

Todorov T., Typologie du roman policier [dans:] idem, Poétique de la prose, Paris 1971.

Tomachevski B., Les genres littéraires [dans:] idem, Théorie de la littérature, Paris 1965.

Tortel J., Le roman populaire [dans:] Entretiens sur la paralittérature, dir. N. Arnaud, F. Lacassin, J. Portel, Cerisy-la-Salle 1970.

Toussaint J.-Ph., L'Autoportrait (à l'étranger), Paris 2000/2012.

Toussaint J.-Ph., L'Urgence et la Patience [dans:] L'Urgence et la Patience, Paris 2012.

Toussaint J.-Ph., La Réticence, Paris 1991.

Toussaint J.-Ph., Le jour ou j'ai commencé La Réticence, «www.bon-a-tirer.com. Revue littéraire en ligne» 2002, no. 6, http://www.bon-a-tirer.com/volume6/jpt. html (accès: 15.02.2019).

Vray J.-B., L'Autoportrait ludique ou le bonnet noir de la mélancolie [dans:] Les vérités de Jean-Philippe Toussaint, dir. S. Chaudier, Saint-Etienne 2016.

Wagner F., Monsieur Jean-Philippe Toussaint et la notion de Vérité. Pour une poétique perspectiviste, «Textyles» 2010, no. 38.

Zawadzki A., Autor. Podmiot literacki [dans:] Kulturowa teoria literatury. Główne pojęcia i problemy, dir. M.P. Markowski, R. Nycz, Kraków 2012. 